\title{
Geopolymer- $\mathrm{TiO}_{2} \mathrm{Nanocomposites} \mathrm{for}$
} Photocsatalysis: Synthesis by One-Step Adding Treatment Versus Two-Step Acidification Calcination

\author{
Xiaohui Yang ${ }^{1}{ }^{D}$, Yi Liu ${ }^{1}$, Chunjie Yan ${ }^{1, *}$, Ronghua Peng ${ }^{2, *}$ and Hongquan Wang ${ }^{1}$ \\ 1 Faculty of Materials Science and Chemistry, China University of Geosciences, Wuhan 430074, China; \\ yxh19512@163.com (X.Y.); yiliu@cug.edu.cn (Y.L.); tang332294@126.com (H.W.) \\ 2 Institute of Geophysics and Geomatics, China University of Geosciences, Wuhan 430074, China \\ * Correspondence: chjyan2005@126.com (C.Y.); pengrh@cug.edu.cn (R.P.); Tel.: +86-2767-885-098 (C.Y.)
}

Received: 15 September 2019; Accepted: 25 October 2019; Published: 27 October 2019

\begin{abstract}
Geopolymer- $\mathrm{TiO}_{2}$ nanocomposites were prepared by two different techniques, namely the two-step acidification calcination treatment and one-step adding method. The potential photocatalytic activities of geopolymer- $\mathrm{TiO}_{2}$ nanocomposites prepared by the two different methods were tested and compared. Nanocomposites prepared via the one-step process showed better photocatalytic activity. The amount of $\mathrm{TiO}_{2}$ particles loaded on the surface of the foaming materials was investigated by XRD and SEM-Mapping. By comparing with the sample obtained from two-step treatment, the $\mathrm{TiO}_{2}$ particles were distributed uniformly on the surface of the foaming materials for the sample obtained from the one-step method in this study. Results showed that the specific surface area of the geopolymer- $\mathrm{TiO}_{2}$ prepared by the one-step treatment process $\left(28.67 \mathrm{~m}^{2} / \mathrm{g}\right)$ was significantly lower than the two-step acidification calcination process $\left(215.04 \mathrm{~m}^{2} / \mathrm{g}\right)$, while the photocatalytic efficiency with methylene blue trihydrate (MB) was better. This is due to the more stable structure of geopolymer- $\mathrm{TiO}_{2}$ nanocomposites, the better dispersion and more loading of $\mathrm{TiO}_{2}$ particles on the foaming materials surfaces, leading to the enhanced photocatalytic activity.
\end{abstract}

Keywords: fly ash; porous materials; $\mathrm{TiO}_{2}$; one-step; photocatalytic; functional

\section{Introduction}

Recently, metal dioxides with excellent photocatalytic activity have been attracting attention [1]. Among the various metal dioxides, $\mathrm{TiO}_{2}$ is usually used as a photocatalyst to treat water pollution for the removal of chemical contaminants [2,3]. Nevertheless, the recovery and the agglomerates of $\mathrm{TiO}_{2}$ nanoparticles are two inevitable problems [4]. Therefore, developing new $\mathrm{TiO}_{2}$ supported catalysts is an urgent problem requiring action.

Coal fly ash (CFA) is generally derived from thermal power generation plants and can negatively impact the environment [5]. Some researchers have concentrated on the preparation of environmentally friendly products from CFA through geopolymerization [6,7]. Geopolymers can be used as supported materials, which not only solves the agglomeration of nanomaterials, but also adsorbs a certain amount of harmful substances [8]. Alouani et. al. removed methylene blue from aqueous solution by using fly ash based geopolymer powder [9]. Further, metakaolin based geopolymer was applied to absorb multiand mono- cations in aqueous solution by Onutai's team [10]. Those studies suggest the possibility of using geopolymer as supports for photocatalyst. In order to increase the amount of the loadable nanoparticles and the contact surface with water, geopolymer could be prepared as a porous structure. However, the existing literature is scarce.

Therefore, we focused on the synthesis of a porous geopolymer supported $\mathrm{TiO}_{2}$ nanocomposite. Two different methods were used in this study. The first one is the one-step method. Geopolymer- $\mathrm{TiO}_{2}$ 
nanocomposites were fabricated by adding $\mathrm{TiO}_{2}$ particles directly during the preparation of the foamed CFA geopolymer. In the two-step method, foamed CFA geopolymer was first prepared and then $\mathrm{TiO}_{2}$ particles were loaded on the surface. In order to explore the relationship between the structural properties of the nanocomposites and the synthesis methods, multiple techniques, including SEM, XRD, and BET were applied to investigate the properties of the geopolymer- $\mathrm{TiO}_{2}$ nanocomposites. The photocatalytic activities of the geopolymer- $\mathrm{TiO}_{2}$ nanocomposites were monitored via testing the degradation time for MB. Therefore, the objective of this work was to investigate if the one-step convenient process could have an obvious effect on enhancing photocatalytic performance by comparing with the two-step method. Although fly ash- $\mathrm{TiO}_{2}$ composites have been investigated by some researchers previously, geopolymer- $\mathrm{TiO}_{2}$ composites were prepared here by the one-step process for the first time, to the best of our knowledge.

\section{Materials and Methods}

CFA was collected from Shenhua Junggar Energy Corporation in Junggar, Inner Mongolia, China, and calcined at $850{ }^{\circ} \mathrm{C}$ for $4 \mathrm{~h}$ before use. Analytical grade sodium hydroxide, $\mathrm{H}_{2} \mathrm{O}_{2}$ solution $(30 \%)$, grade oleic acid, $\mathrm{TiO}_{2}(\mathrm{P} 25)$, methylene blue trihydrate, and nitric acid $\left(\mathrm{HNO}_{3}\right)$ were purchased from Sinopharm Chemical Reagent Co., Ltd. Commercial sodium water glass with original modulus of 3.2 was purchased from Guangdong Foshan Zhongfa Co., Ltd., China, and deionized water is self-made in the laboratory.

The foam materials were prepared based on our previous literature [11]. The alkaline activator (the modulus is 1.2) was thoroughly stirred at $1800 \mathrm{rpm}$ for $8 \mathrm{~min}$. Then CFA (50 g) was mixed at $1800 \mathrm{rpm}$ for $5-6 \mathrm{~min}$ after adding to the activator solution. Oleic acid $(0.3 \mathrm{~g})$ was added and mixed for another 3-5 min, then $\mathrm{H}_{2} \mathrm{O}_{2}$ solution $(2.25 \mathrm{~mL})$ was added to the mixture, stirring at $2000 \mathrm{rpm}$ for 3-5 min to make geopolymer foam. Finally, the foam mixture was cast into a silica gel mold and cured at $80^{\circ} \mathrm{C}$ for $24 \mathrm{~h}$. The material was named as GF.

\subsection{Preparation of Geopolymer-Supported $\mathrm{TiO}_{2}$ by One-Step Process}

Subsequently, $5 \mathrm{~g} \mathrm{TiO}_{2}(10$ mass $\%$ ) was added to the foamed geopolymer paste, and stirred for further 3-5 $\mathrm{min}$. The final product was named as GFT1.

\subsection{Preparation of Geopolymer-Supported $\mathrm{TiO}_{2}$ by Two-Steps Process}

As specified in previous studies [12], $3.0 \mathrm{~g} \mathrm{TiO}$ 2 was dissolved in $100 \mathrm{~mL} \mathrm{HNO}_{3}(1 \mathrm{M}), 500 \mathrm{~mL}$ deionized water was then added and stirred an hour under the condition of $70{ }^{\circ} \mathrm{C}$. Then $10 \mathrm{~g}$ geopolymer was added to the solution and stirred for about $1 \mathrm{~h}$. The materials were washed four times with deionized water for removing excess unloaded $\mathrm{TiO}_{2}$ and calcined at $500{ }^{\circ} \mathrm{C}$ for $2 \mathrm{~h}$, gradually. The product was named as GFT2.

X-ray powder diffraction (XRD) (D8-FOCUS, BrokerAXS (Beijing) Technology Co., Ltd., Beijing, $\mathrm{China}, \mathrm{Cu}-\mathrm{K} \alpha$ radiation) was used to measure the crystalline phases. The surface morphology of geopolymer- $\mathrm{TiO}_{2}$ composites was characterized by scanning electron microscope (SEM, Hitachi SU8010, Hitachi, Tokyo, Japan). The measurements of specific surface area and pore volume were carried out via measuring $\mathrm{N}_{2}$ adsorption-desorption isotherms at $77 \mathrm{~K}$ using a Micromeritics ASAP 2020 apparatus (Micromeritics Instrument (shanghai) Ltd., Shanghai, China). Photocatalytic activity was tested using a $300 \mathrm{~W}$ Xe lamp (CEL-HXF300, Beijing Zhongjiao Jinyuan Technology Co., Ltd., Beijing, China). $0.04 \mathrm{mg}$ catalyst synthesized by one-step method and by two step process was separately adjunct to MB solution (50 mL) of $10 \mathrm{mg} / \mathrm{L}$ concentration. The mixture was stirred in dark condition for $30 \mathrm{~min}$ in order to reduce the error caused by adsorption-desorption. After irradiation, the degradation of MB was measured by a UV/vis spectrometer (Lambda 35, Perkinelmer, Guangzhou, China) at $665 \mathrm{~nm}$, which was calculated to concentration by the Beer-Lambert law. 


\section{Results and Discussion}

\subsection{XRD Analysis}

Figure 1 shows the XRD patterns of the geopolymer and the geopolymer- $\mathrm{TiO}_{2}$ composites synthesized by different preparation approaches. The geopolymer (GF) owns one "hump" located at around $26.0-28.0^{\circ}(2 \theta)$. For GFT1, the diffraction peak of the anatase phase of titania appears at $2 \theta=25.3^{\circ}$ (JCPDS NO. 65-5714), and the other diffraction peak is ascribed to the faujasite zeolite (JCPDS NO. 26-0897). Compared to GFT2, the anatase phase of titania exhibits greater sharpness and more intensity (101) of reflection in the GFT1 sample. A part of $\mathrm{TiO}_{2}$ was lost in the two-step method during the activation process of $\mathrm{TiO}_{2}$ in dilute acid solution, while one-step method could avoid the waste of $\mathrm{TiO}_{2}$ on the surface of the support material. This means that the one-step method is more conductive to the preparation of the geopolymer- $\mathrm{TiO}_{2}$ composite.

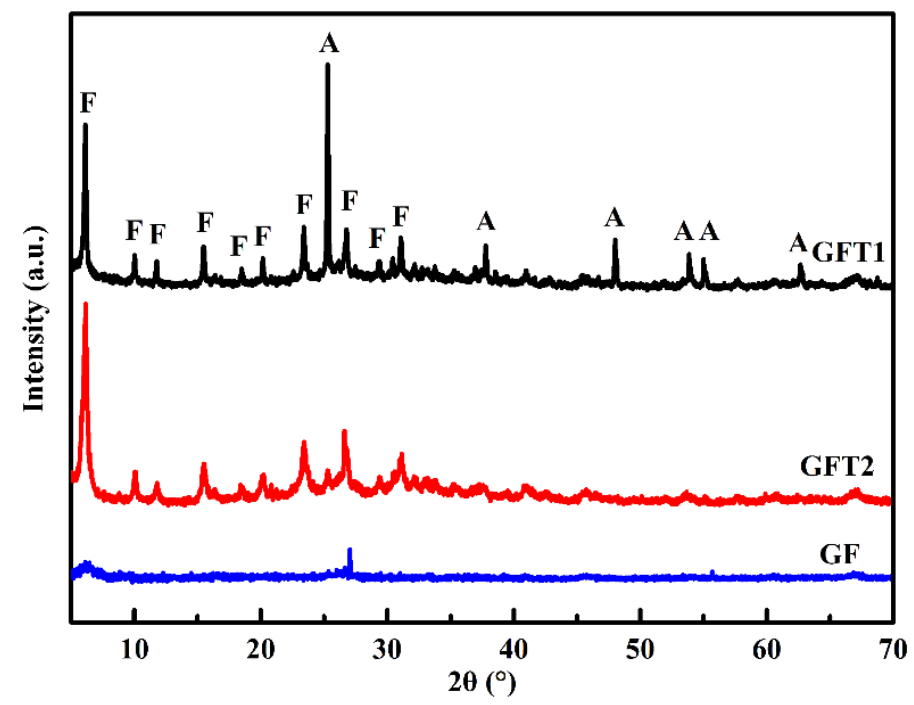

Figure 1. XRD patterns of the geopolymer (GF) and the geopolymer- $\mathrm{TiO}_{2}$ composites obtained by two different approach. A-anatase, F-faujasite.

\subsection{SEM Observations}

The surface morphology of GF without any $\mathrm{TiO}_{2}$ (Figure 2a,b) and GFT1 (Figure 2c,d) or GFT2 (Figure 2e,f) are presented in Figure 2. From Figure 2a, the highly interconnected pores are observed on the surface of the geopolymer, and the material contains 45.91 mass $\%$ O, $10.51 \mathrm{mass} \% \mathrm{Na}, 25.44 \mathrm{mass} \%$ $\mathrm{Al}$, and 18.13 mass\% Si (Figure $2 \mathrm{~b}$ ). Figure 2c-f indicates morphological changes and distribution of Ti in composites with the use of different preparation treatments. The SEM images of GFT1 (Figure 2c) reveal that the excellent porous material formed by the uniform combination of $\mathrm{TiO}_{2}$ particles and CFA could be observed. From SEM elemental mapping images (Figure 2d), well-dispersed $\mathrm{TiO}_{2}$ particles (about 6.63 mass \%) throughout the composite samples is related to the one-step method [13]. The porous material with a smoother surface and a more uniform pore is shown in Figure $2 \mathrm{e}$, and a small amount of $\mathrm{TiO}_{2}$ (3.63 mass\%, about half of one-step method) is supported on the surface of the porous material by the two step preparation process, which could be reflected by SEM elemental mapping images (Figure 2f). Different treatment methods caused not only a difference in the morphology of the composite materials, but also the amounts and the extent of dispersion of $\mathrm{TiO}_{2}$ in the composite, which is consistent with XRD analysis. 

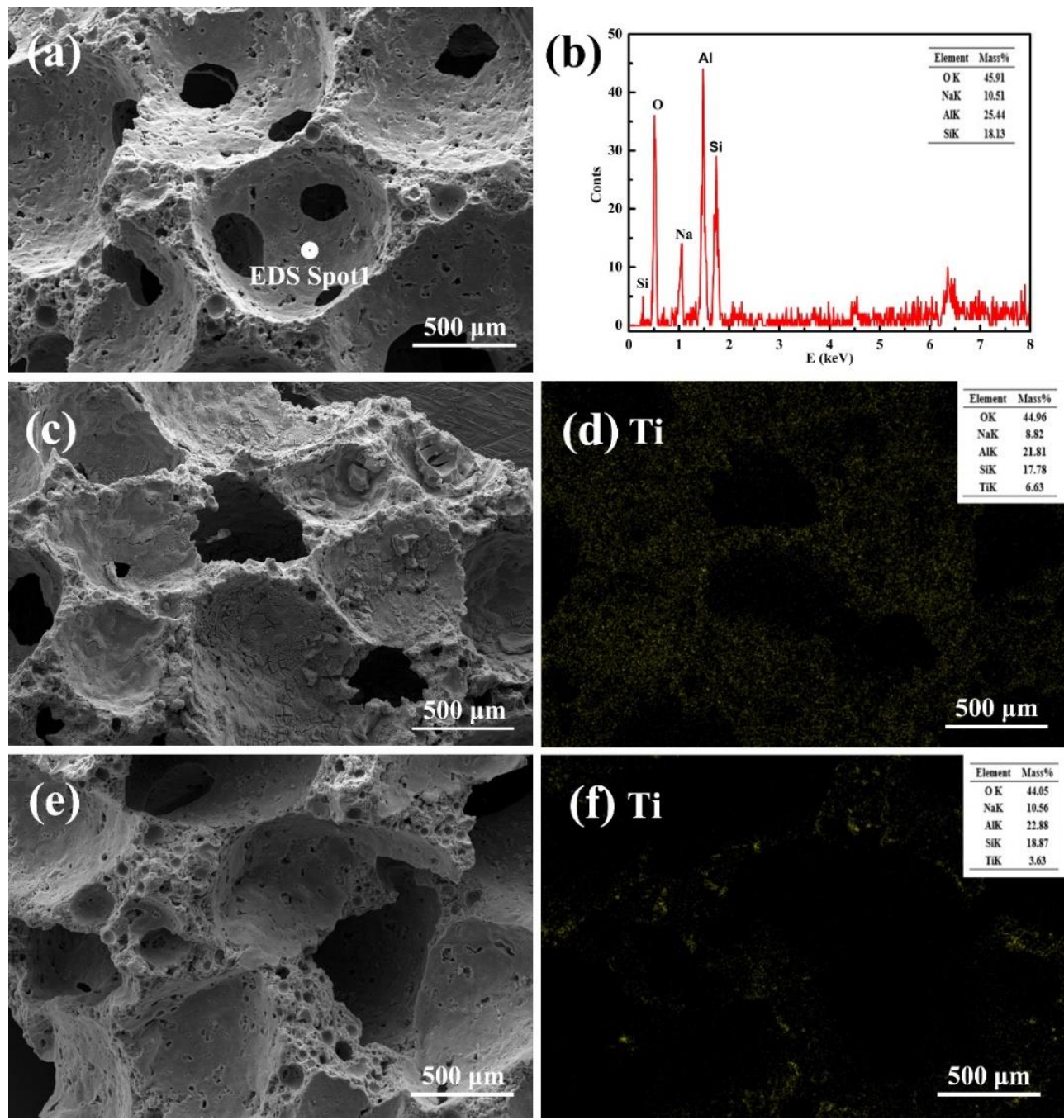

Figure 2. SEM images of geopolymer and geopolymer- $\mathrm{TiO}_{2}$ composite synthesized via two methods:

(a) GF, (b) EDS spectra of GF, (c) GFT1, (d) Ti-mapping of GFT1, (e) GFT2 and (f) Ti-mapping of GFT2.

\subsection{BET Analysis}

Table 1 summarizes the pore structure of GFT1 and GFT2. By comparing with GFT1, the total pore volume of GFT2 increases from 0.13 to $0.26 \mathrm{~cm}^{3} / \mathrm{g}$, the $t$-plot micropore area increases from 0 to $135.39 \mathrm{~m}^{2} / \mathrm{g}$ and the BET surface area increases from 28.67 to $215.04 \mathrm{~m}^{2} / \mathrm{g}$. GFT1 synthesized by the one-step method has a BET surface area of $28.67 \mathrm{~m}^{2} / \mathrm{g}$, which is significantly lower than GFT2. The $t$-plot micropore area for GFT1 is 0 and the total pore volume is only half of GFT2. This phenomenon is caused by the well-distribution of $\mathrm{TiO}_{2}$ nanoparticles for sample GFT1, which is in favor of improving the photocatalytic efficiency.

Table 1. Comparison of product properties between GFT1 and GFT2.

\begin{tabular}{cccc}
\hline \multirow{2}{*}{ Sample } & \multicolumn{2}{c}{ Surface Area $\left(\mathrm{m}^{2} / \mathrm{g}\right)$} & \multirow{2}{*}{$V_{\text {Total }}\left(\mathrm{cm}^{3} / \mathrm{g}\right)$} \\
\cline { 2 - 3 } & $S_{\text {BET }}$ & $S_{t \text {-plot }}$ & \\
\hline GFT1 & 28.67 & 0 & 0.13 \\
GFT2 & 215.04 & 135.39 & 0.26 \\
\hline
\end{tabular}

$V$ total, total pore volume; St-plot, $t$-plot micropore area; $S_{\mathrm{BET}}$, BET surface area. 


\subsection{Photocatalytic Activity}

For exploring the influence of geopolymer and geopolymer- $\mathrm{TiO}_{2}$ composites prepared by two methods on the photocatalytic efficiency, the photocatalytic degradation performance of MB is compared. As shown in Figure 3, GFT1 synthesized by one-step convenient method exhibited better photocatalytic efficiency for MB. The degradation is completed in ca. $15 \mathrm{~min}$. However, GFT2 synthesized by the two-step method presented lower activity than GFT1. The MB is degraded after $45 \mathrm{~min}$ of irradiation. Although the photocatalysis property of geopolymer without any $\mathrm{TiO}_{2}$ particles (GF) is relatively low, the material could fully degrade MB after $50 \mathrm{~min}$. This may be caused by the high adsorption capacity of the geopolymer and some precious metals that have photocatalytic effect contained in the geopolymer [11,14]. The lower amount of anatase phase of $\mathrm{TiO}_{2}$ (Figure 1), and uneven dispersion of $\mathrm{TiO}_{2}$ on the surface of the GFT1 sample (Figure 2) suggest that the GFT1 sample could be a potential candidate for the treatment of industrially discharged wastewater.

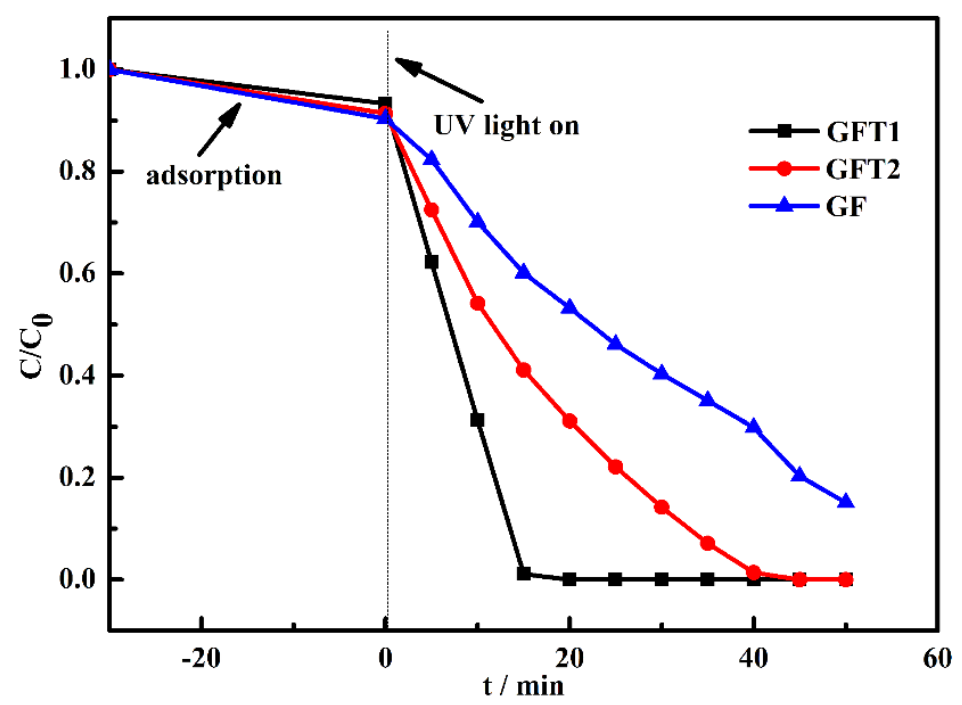

Figure 3. Change in $\mathrm{MB}$ concentration versus irradiation time of geopolymer and geopolymer- $\mathrm{TiO}_{2}$ composites obtained by two preparation approach.

\section{Conclusions}

In summary, the geopolymer- $\mathrm{TiO}_{2}$ nanocomposites were prepared via two different processes, namely the one-step method and two-step treatment. The preparation process was found to significantly affect the $\mathrm{TiO}_{2}$ loading amount, dispense extent, porosity, and surface area of the resultant products. $\mathrm{TiO}_{2}$ can be uniformly distributed under the condition of high-speed stirring by the one-step process. In addition, the one-step method avoided the waste of $\mathrm{TiO}_{2}$, and increased the loading amount of the composites. Composites prepared by the one-step method were superior to those synthesized by the two-step method for not only improving the crystallite loading and distribution, but also enhancing the photocatalytic performance.

Author Contributions: conceptualization, Y.L.; formal analysis, H.W.; resources, C.Y. and R.P.; writing-original draft preparation-review and editing, X.Y.

Funding: The Project was Supported by the Fundamental Research Funds for the Central Universities, China University of Geosciences (Wuhan) (grant: 26420190057) and Engineering Research Center of Nano-Geo Materials of Ministry of Education, China University of Geosciences (Wuhan) (grant: NGM2019KF016).

Conflicts of Interest: The authors declare no conflict of interest. 


\section{References}

1. Zhou, W.; Pang, Y.; Lai, S.; Yang, Z. Fabrication of $\mathrm{TiO}_{2}$ nanoparticles loaded on coal fly ash composite with enhanced photocatalytic activity. J. Nanosci. Nanotechnol. 2012, 12, 4658-4663. [CrossRef] [PubMed]

2. Zhou, X.-T.; Ji, H.-B.; Huang, X.-J. Photocatalytic Degradation of Methyl Orange over Metalloporphyrins Supported on $\mathrm{TiO}_{2}$ Degussa P25. Molecules 2012, 17, 1149-1158. [CrossRef] [PubMed]

3. Sun, Z.; Yuan, F.; Li, X.; Li, C.; Xu, J.; Wang, B. Fabrication of Novel Cyanuric Acid Modified g- $\mathrm{C}_{3} \mathrm{~N}_{4} / \mathrm{Kaolinite}$ Composite with Enhanced Visible Light-Driven Photocatalytic Activity. Minerals 2018, 8, 437. [CrossRef]

4. Zhou, F.; Yan, C.; Wang, H.; Zhou, S.; Komarneni, S. Sepiolite-TiO 2 Nanocomposites for Photocatalysis: Synthesis by Microwave Hydrothermal Treatment Versus Calcination. Appl. Clay Sci. 2017, 146, $246-253$. [CrossRef]

5. Wang, B.; Zhou, Y.; Li, L.; Xu, H.; Sun, Y.; Du, Y.; Wang, Y. In Situ Synthesis of $\mathrm{TiO}_{2}$-Doped Mesoporous Silica from Coal Fly Ash for the Photocatalytic Degradation of Dyes. Ind. Eng. Chem. Res. 2018, 57, 15632-15637. [CrossRef]

6. Katančić, Z.; Gavran, I.; Smolković, J.; Hrnjak-Murgić, Z. Fly Ash Supported Photocatalytic Nanocomposite Poly(3,4-Ethylenedioxythiophene)/ $/ \mathrm{TiO}_{2}$ for Azo Dye Removal under Simulated Solar Irradiation. J. Appl. Polym. Sci. 2018, 135, 46316. [CrossRef]

7. Singh, N.B. Fly Ash-Based Geopolymer Binder: A Future Construction Material. Minerals 2018, 8, 299. [CrossRef]

8. Visa, M.; Andronic, L.; Duta, A. Fly Ash-TiO 2 Nanocomposite Material for Multi-Pollutants Wastewater Treatment. J. Environ. Manag. 2015, 150, 336-343. [CrossRef] [PubMed]

9. El Alouani, M.; Alehyen, S.; El Achouri, M.; Taibi, M. Removal of Cationic Dye-Methylene Blue-From Aque Ous Solution by Adsorption on Fly Ash-Based Geopolymer. J. Mater. Environ. Sci. 2018, 9, $32-46$.

10. Onutai, S.; Kobayashi, T.; Thavorniti, P.; Jiemsirilers, S. Metakaolin Based Geopolymer from Thailand as an Adsorbent for Adsorption of Multi- and Mono- Cations from Aqueous Solution. Key Eng. Mater. 2018, 777, 245-250. [CrossRef]

11. Liu, Y.; Yan, C.; Zhang, Z.; Gong, Y.; Wang, H.; Qiu, X. A facile method for preparation of floatable and permeable fly ash-based geopolymer block. Mater. Lett. 2016, 185, 370-373. [CrossRef]

12. Uğurlu, M.; Karaoğlu, M.H. Photocatalytic Removal of Olive Mill Waste Water by $\mathrm{TiO}_{2}$ Loaded on Sepiolite and under Natural Solar Irradiation. Environ. Prog. Sustain. Energy 2011, 30, 326-336. [CrossRef]

13. Weng, C.-J.; Huang, J.-Y.; Huang, K.-Y.; Jhuo, Y.-S.; Tsai, M.-H.; Yeh, J.-M. Advanced Anticorrosive Coatings Prepared from Electroactive Polyimide- $\mathrm{TiO}_{2}$ Hybrid Nanocomposite Materials. Electrochim. Acta 2010, 55, 8430-8438. [CrossRef]

14. Zhang, Y.; Liu, L. Fly ash-based geopolymer as a novel photocatalyst for degradation of dye from wastewater. Particuology 2013, 11, 353-358. [CrossRef]

(C) 2019 by the authors. Licensee MDPI, Basel, Switzerland. This article is an open access article distributed under the terms and conditions of the Creative Commons Attribution (CC BY) license (http://creativecommons.org/licenses/by/4.0/). 